\title{
AVERSIÓN AL RIESGO, PREFERENCIA TEMPORAL Y VARIABLES SOCIOECONÓMICAS. EVIDENCIA DE UN PUEBLO DE COLOMBIA
}

David Ortiz Escobar*

$\mathrm{E}$ ste artículo presenta evidencia exploratoria sobre la asociación entre ciertas variables socioeconómicas e indicadores de preferencias individuales en Colombia. Explora la relación entre edad, nivel educativo y exposición al conflicto armado, por un lado, y aversión al riesgo y preferencia temporal, por el otro. Los indicadores de preferencias individuales se obtuvieron a partir de experimentos de campo, mediante encuestas incentivadas.

Desde hace varias décadas, la economía experimental viene refinando un conjunto de protocolos para obtener indicadores de preferencias temporales (Harrison et al., 2002), aversión al riesgo (Charness et al., 2013), confianza interpersonal (Berg et al., 1995), honestidad (Fischbacher, 2013) o preferencias pro sociales (Fehr y Gächter, 2000) en diferentes grupos poblacionales. Los participantes en los experimentos deben tomar decisiones -en un contexto de incentivos reales- que revelan sus preferencias individuales ${ }^{1}$.

De acuerdo con la clasificación de Harrison y List (2004), en experimentos de campo -así como en los experimentos de laboratorio- el investigador diseña un conjunto artificial de reglas e incentivos que comunica a los participantes en un lenguaje abstracto y descontex-

* Doctor en Economía, profesor e investigador, Facultad de Economía, Universidad Externado de Colombia, Bogotá, [david.ortiz@uexternado.edu.co]. Agradezco a la Fundación Guaicaramo por financiar la investigación y autorizar la divulgación de los resultados, y a los miembros del equipo: Andrea Beltrán, María Elisa Balen, Ana María Pedraza, Melissa Guerrero, Erika Rodríguez y Nicolás Pulido por sus opiniones y su ayuda en el proyecto. Fecha de recepción: 27-09-2016, fecha de modificación: 23-06-2017, fecha de aceptación: 04-09-2017. Sugerencia de citación: Ortiz E., D. (2017). Aversión al riesgo, preferencia temporal y variables socioeconómicas. Evidencia de un pueblo de Colombia, Revista de Economía Institucional 19(37), 147-165. Dor: https://doi.org/10.18601/01245996.v19n37.08

${ }_{1}$ Algunos estudios emplean protocolos de revelación de preferencias individuales con preguntas hipotéticas, es decir, sin incentivos reales; ver Johnson (2002). 
tualizado ${ }^{2}$. La principal diferencia radica en que en los experimentos de campo los participantes se toman de una población no estándar, es decir, no limitada a estudiantes universitarios.

Los experimentos de campo presentan grandes retos. Es necesario adaptar instrumentos experimentales tradicionalmente aplicados a la población universitaria, a personas con niveles más bajos de razonamiento abstracto. $\mathrm{E} 1$ reclutamiento de los participantes, además de ser más dispendioso que en un campus universitario, conlleva sesgos de selección importantes. Como anotan Harrison y List (2004), “existe selección de la muestra en dos etapas [...] El individuo selecciona estar en el ambiente natural [en este caso, en el municipio donde se hizo el estudio] y luego tomar la decisión de participar en el experimento". Por tanto, aunque se trata de experimentos con "personas reales", no necesariamente exhiben mayor validez externa que experimentos con estudiantes universitarios.

No obstante, dado el objetivo de este artículo, tales experimentos tienen una ventaja sobre los experimentos de laboratorio tradicionales: la variabilidad de la muestra en ciertos factores de interés como la edad, el nivel de ingresos o la exposición al conflicto armado, que a su vez permite determinar con mayor precisión la asociación entre estas variables y las preferencias individuales ${ }^{3}$.

La variabilidad de la muestra, sin embargo, no soluciona el problema de la causalidad. Recientemente, varios estudios han tratado de ir más allá de la simple asociación entre las preferencias individuales y las condiciones socioeconómicas u otras experiencias personales, y han propuesto estrategias empíricas para aislar los efectos causales entre estas variables. Podemos dividir esta literatura en dos grupos: el objetivo del primer grupo es establecer los efectos de las preferencias individuales sobre variables socioeconómicas, mientras que el del segundo grupo es explorar el efecto de ciertas circunstancias individuales en las preferencias temporales, la aversión al riesgo y las preferencias pro sociales.

El primer objetivo es relevante debido a que la aversión al riesgo y la preferencia temporal son determinantes de las decisiones de ahorro e inversión. Una pregunta empírica fundamental es en qué medida las discrepancias socioeconómicas reflejan diferencias en las prefe-

\footnotetext{
2 Aunque esta es la práctica general, algunos autores investigan los efectos de introducir un contexto explícito en las instrucciones sobre las decisiones de los jugadores; ver, p. ej., Dufwenberg et al. (2011).

${ }^{3}$ Harrison y List (2004) indican: “El problema con los estudiantes' es la falta de variabilidad de sus características socio-demográficas, no la falta de representatividad de sus respuestas condicionadas a sus características socio-demográficas".
} 
rencias individuales, o si se deben más bien a factores institucionales (Cárdenas et al., 2008). Otros autores han ahondado en los efectos de los niveles de aversión al riesgo y preferencia temporal en aspectos como logros educativos y variables relacionadas con la salud (Castillo et al., 2011; Sutter et al., 2013). El segundo grupo de literatura ha implementado estrategias empíricas para establecer cuál es el efecto causal de factores como la educación o la exposición al conflicto armado sobre las preferencias individuales (Callen et al., 2014; Voors et al., 2012). Esto es relevante pues las políticas económicas deben tener en cuenta los perfiles específicos de grupos de población que históricamente han tenido menos acceso a servicios de educación y salud, o que han estado más expuestos a los conflictos armados, la violencia y el despojo de tierras.

Este estudio es parte del proyecto de caracterización socioeconómica de Barranca de Upía realizado en febrero de 2015 y financiado por la Fundación Guaicaramo. Barranca de Upía es un pequeño pueblo del departamento del Meta que ha recibido inmigrantes de todas las regiones, tanto por desplazamiento violento como por motivos económicos. El pueblo ha pasado por diferentes fases de desarrollo económico, en las que la agroindustria y las actividades extractivas han jugado un papel preponderante.

El eje central del proyecto de caracterización fue una encuesta a 370 hogares. A una sub muestra de los encuestados le aplicamos un cuestionario incentivado, cuyo objetivo era obtener indicadores de las preferencias económicas individuales. El cruce de la información recolectada con los dos instrumentos nos da luces sobre las asociaciones existentes entre preferencia temporal, aversión al riesgo y variables socioeconómicas. Aunque la estrategia empírica no permite aislar efectos causales, los resultados señalan avenidas de investigación relevantes en un contexto de post conflicto, especialmente en aspectos relacionados con las preferencias económicas de la población desplazada.

En resumen, el análisis de los resultados muestra que 1) la edad está asociada a la preferencia temporal revelada, pero no a la aversión al riesgo revelada; 2) las mujeres son más aversas al riesgo, y 3) el hecho de haber llegado al pueblo en los últimos cinco años, bien sea buscando oportunidades económicas o huyendo del conflicto armado, está asociado a una alta preferencia temporal revelada y una baja aversión al riesgo revelada. 


\section{PROCEDIMIENTOS}

\section{SELECCIÓN DE PARTICIPANTES DENTRO DE LA ENCUESTA A HOGARES}

Realizamos la encuesta de hogares del 12 al 22 de febrero de 2015, con un equipo de cinco encuestadores debidamente entrenados. E1 cuestionario abarcó diversos temas sobre calidad de vida y percepciones generales de los barranqueños ${ }^{4}$. Aunque también hicimos encuestas en la zona rural, solo aplicamos el cuestionario para obtener preferencias (en adelante, cuestionario específico) a una sub muestra de habitantes de la cabecera municipal.

Una vez finalizada la encuesta, el encuestado era invitado a una actividad en las instalaciones de la Fundación Guaicaramo si sabía leer y escribir. Los encuestadores no usaron palabras técnicas: se referían a la actividad como un espacio donde los participantes responderían otras preguntas y participarían en juegos en los que se podía ganar premios en dinero. Por la asistencia ofrecimos $\$ 5.000$ en efectivo (unos 2,5 dólares) y un refrigerio, a fin de remunerar el desplazamiento a las instalaciones y el tiempo que dedicarían a la actividad ${ }^{5}$. Para facilitar la asistencia hicimos una lista de horarios en los que los interesados podían inscribirse según sus posibilidades.

Administramos el cuestionario específico a 48 mujeres y 20 hombres distribuidos en varios grupos. Los participantes se identificaban con el consecutivo de la encuesta de hogares, lo que nos permitiría cruzar la información obtenida en la misma con los resultados del cuestionario específico. Cada persona llenaba su formulario individualmente después de la explicación a cada pregunta. Los participantes podían pedir aclaraciones solo al líder de la actividad y desde el principio solicitamos que los asistentes no hablaran entre sí.

\section{MÉTODOS PARA OBTENER PREFERENCIAS}

Existen diversos métodos para obtener indicadores individuales de la preferencia temporal y la aversión al riesgo. Los investigadores han ido adaptándolos al ritmo de las innovaciones teóricas en estos campos (Frederick et al., 2002; Charness et al., 2013). Generalmente los instrumentos son aplicados a estudiantes universitarios que constituyen un grupo poblacional especial. El reto de crear instrumentos

${ }^{4}$ Los pormenores del procedimiento de selección aleatoria de hogares para realizar la encuesta están disponibles a petición de los interesados.

${ }^{5}$ En las reuniones con el grupo investigador concluimos que el efecto de estos incentivos sobre la participación es ambiguo, en especial si se trata de dinero en efectivo. Aunque el reconocimiento en dinero motivó a muchas personas a participar en la actividad, algunos encuestados vieron con sospecha este incentivo. 
para obtener preferencias individuales radica entonces en adaptar los ya existentes para que sean aplicados a un grupo más representativo de la población.

Un problema frecuente en este tipo de experimentos es el nivel de habilidad numérica de los participantes, que en nuestro caso es más bajo que el de los estudiantes universitarios. Chetan et al. (2010) concluyeron al respecto que cuando los individuos exhiben baja habilidad aritmética es preferible usar instrumentos simples con los que podemos obtener indicadores de las preferencias poco ruidosos y con un buen poder predictivo. Por lo tanto, en este estudio seguimos la recomendación de Cárdenas et al. (2008) de usar "instrucciones simples que se basan en imágenes, diagramas y ejemplos [...] más que en textos y funciones de pago complejas".

El instrumento tenía tres partes: un cuestionario general, un cuestionario sobre preferencias temporales y un cuestionario sobre aversión al riesgo. Para hacer el experimento compatible con los incentivos monetarios, al final de cada sesión escogimos una o dos personas al azar-dependiendo del tamaño del grupo- para hacer efectiva una de las partes del cuestionario. Con el fin de elevar la credibilidad de la actividad, aclaramos a los participantes que los pagos futuros estaban garantizados por la Fundación Guaicaramo.

\section{PREFERENCIAS TEMPORALES}

Para obtener preferencias temporales usamos el método clásico de la lista múltiple de precios (MPL) popularizada por Harrison et al. (2002). La pregunta modelo fue: “ $¿ Q u e ́$ preferiría: recibir $\$ 10.000$ mañana o recibir $\$ 10.000+x$ pesos de mañana en una semana?”. Donde $x$ variaba de 0 a 9.000 en múltiplos de 1.000 , de tal forma que en la primera línea los participantes debían escoger entre $\$ 10.000$ mañana y $\$ 10.000$ de mañana en una semana, mientras que la última línea presentaba la opción de $\$ 10.000$ mañana y $\$ 19.000$ de mañana en una semana (ver anexo 1). \$10.000 (5 dólares) es una cantidad interesante pues equivalen aproximadamente a medio jornal de trabajo.

En nuestra lista múltiple de precios la primera opción de cada línea era siempre recibir $\$ 10.000$, no el mismo día del experimento sino al día siguiente, para neutralizar cualquier ruido que pudieran causar los costos de transacción y los problemas de confianza. Como las personas favorecidas por el sorteo tenían que volver a las oficinas de la Fundación por su premio sin importar su decisión, los factores mencionados no producían un sesgo a favor de la decisión más cercana. De esta manera nos acercábamos al objetivo de la actividad: que los 
participantes imaginaran un problema lo más parecido a la elección abstracta entre dos cantidades de dinero en el tiempo.

El ejercicio fue diseñado para que los participantes siempre escogieran la opción más cercana en la primera línea, ya que no había ninguna compensación por esperar una semana. Sin embargo, a medida que avanzaba el cuestionario, la decisión de esperar se volvía más atractiva. $\mathrm{El}$ indicador de la preferencia temporal es precisamente la línea en la que el participante decide esperar una semana a cambio de un incremento en su pago. Las tasas de interés nominales anuales asociadas a estas decisiones son bastante altas (de 0 a $4.600 \%)^{6}$ y el cuestionario produce datos censurados sobre las tasas de descuento individuales. A pesar de lo anterior, en este estudio estamos interesados en indicadores ordinales de la preferencia temporal ${ }^{7}$.

\section{AVERSIÓn AL RIESGO}

Para obtener medidas de aversión al riesgo seguimos el método propuesto por Cárdenas y Carpenter (2013). Los participantes eligieron una de seis loterías binarias ${ }^{8}$, en las que la probabilidad de cada pago era igual. Para seleccionar el pago pusimos una pelota negra y una pelota blanca en una bolsa, y sacábamos una al azar. La primera lotería representaba un pago fijo de $\$ 7.000$ (3,5 dólares); el participante ganaría esta suma sin importar qué pelota saliera de la bolsa. A partir de la segunda lotería, la diferencia de los pagos empezaba a aumentar hasta llegar al sexto juego, en el que la pelota blanca ofrecía un pago de $\$ 0$ y la pelota negra uno de $\$ 20.000$ (10 dólares) (ver anexo 2).

Siguiendo a Cárdenas y Carpenter, para determinar qué implica cada una de esas decisiones en términos de aversión al riesgo, partimos de una función tipo CRRA, donde $r$ representa el coeficiente relativo de aversión al riesgo:

${ }^{6}$ La tasa de interés nominal anual que implica cada decisión se calculó así: [(Monto $2-$ Monto $\left._{1}\right) /$ Monto $\left._{1}\right] \times 52$, donde Monto 2 es la cantidad ofrecida por esperar una semana, Monto ${ }_{1}$ el premio entregado si el individuo decide no esperar una semana. Todo se multiplica por 52, el número de semanas del año.

7 Este instrumento no capta anomalías como el descuento hiperbólico o cuasihiperbólico y, por tanto, no permite detectar el grado de inconsistencia dinámica de las preferencias individuales. En el descuento hiperbólico la tasa de descuento aumenta cuanto más cercano es el punto de referencia de la decisión. Por ejemplo, individuos que preferirían $x$ hoy a $x+1$ dentro de un mes, podrían preferir $x+1$ dentro de un año y un mes a $x$ dentro de un mes, revelando un sesgo hacia la satisfacción presente. Kirby y Merrnstein (1995) presentan evidencia experimental a este respecto.

${ }^{8}$ En la prueba piloto encontramos que el concepto de "lotería" puede tener una representación negativa, especialmente entre los grupos cristianos que constituyen un buen porcentaje de los habitantes del pueblo. Por tanto, los sustituimos por "juego". 
$U(x)=x^{1-\mathrm{r}} /(1-r)$

Un individuo sería indiferente entre cualquier par de loterías si

$0,5 U\left(x_{1} a\right)+0,5 U\left(x_{1} b\right)=0,5 U\left(x_{2} a\right)+0,5 U\left(x_{2} b\right)$

donde 1 y 2 son las loterías binarias y a y $b$ representan cada uno de los pagos. Al resolver estas ecuaciones para nuestras loterías obtenemos los coeficientes de aversión al riesgo resumidos en el cuadro 1.

Cuadro 1

Rangos del coeficiente de aversión al riesgo según las loterías (Pagos en pesos)

\begin{tabular}{crr}
\hline & Pagos & Valores de $r$ \\
\hline Lotería 1 & 7.000 & $r>2,32$ \\
\hline Lotería 2 & 7.000 & 2.500 \\
\hline Lotería 3 & 10.000 & $2,32>r>0,781$ \\
\hline Lotería 4 & 13.000 & $0,781>r>0,556$ \\
\hline Lotería 5 & 16.500 & $0,556>r>0,216$ \\
\hline Lotería 6 & 1.000 & $0,216>r>0$ \\
\hline Lute: & 20.000 & $r<0$ \\
\hline
\end{tabular}

Fuente: elaboración propia.

Si el individuo escoge una lotería de la 1 a la 5 , revela que es averso al riesgo; si elige la última, revela que es amante del riesgo. Como advertí en la sección de preferencias temporales, el cuestionario arroja datos censurados y no es posible determinar un coeficiente de aversión al riesgo a nivel individual. Sin embargo, en este estudio estamos interesados en obtener indicadores ordinales que capten las diferencias interpersonales en cuanto a sus preferencias por el riesgo'.

\section{RESULTADOS}

\section{PERFIL DE QUIENES RESPONDIERON EL CUESTIONARIO ESPECÍFICO}

E1 cuadro 2 resume algunas características de los participantes de las sesiones experimentales y las compara con las características promedio de la población de Barranca de Upía (según la encuesta de hogares). En términos generales los participantes del experimento son mayores

9 Por tanto, el instrumento solo capta la aversión al riesgo en las ganancias. De acuerdo con la teoría de la prospección (Kahneman y Tversky, 1979), el resultado puede ser diferente si los individuos enfrentan pérdidas. Ver Cárdenas y Carpenter (2013) para un instrumento sencillo que captaría aversión al riesgo en las pérdidas. 
y un poco más educados que la población total.Además, hay una sobre representación de las mujeres.

Cuadro 2

Características de los encuestados y los participantes en el experimento

\begin{tabular}{lcc}
\hline & Encuesta & Experimento \\
\hline Total muestra & $\begin{array}{c}370 \text { hogares, } \\
1.403 \text { personas }\end{array}$ & 68 personas \\
\hline Sexo & $50 \%$ & $30 \%$ \\
Masculino & $50 \%$ & $70 \%$ \\
Femenino & & \\
\hline Edad & 28 & 40 \\
Media & 24 & 40 \\
Mediana & & \\
Nivel educativo & $23 \%$ & $32 \%$ \\
Terminó bachillerato & $77 \%$ & $68 \%$ \\
No terminó bachillerato & & \\
El jefe de hogar cambió de residencia en los últimos 5 años & $25 \%$ & $30 \%$ \\
Sí & $75 \%$ & $70 \%$ \\
No &
\end{tabular}

Fuente: elaboración propia.

\section{PREFERENCIATEMPORAL}

\section{ELECCIONES INCONSISTENTES O REVERSIONES}

Si un individuo expresa que preferiría $\$ 10.000+x$ en una fecha lejana a \$10.000 en una fecha cercana, ha revelado información sobre su tasa de descuento temporal. Las preferencias son consistentes si para cada $x^{\prime}>x$ el individuo siempre prefiere el pago en la fecha lejana al pago en la fecha cercana. En la lista de decisiones de nuestro experimento, una reversión de preferencias se da cuando el participante elige en una línea el premio en la fecha lejana y en una línea posterior elige el premio en la fecha cercana. Varios factores explican esta conducta: falta de habilidad aritmética, no haber comprendido bien las instrucciones del juego o el uso de heurísticas de decisión que se alejan de los axiomas de las preferencias reveladas.

De los participantes, 12 presentaron algún tipo de inconsistencia en su elección temporal ( $18 \%$ de la muestra). El cuadro 3 resume las variables que posiblemente están correlacionadas con elecciones inconsistentes durante el experimento. E1 24\% de las personas que no terminaron bachillerato tomó alguna decisión inconsistente, mientras que solo lo hizo el 5\% de las personas que terminaron educación secundaria $(\mathrm{p}(\mathrm{Z}>\mathrm{z})=0,023)$. E1 32\% de los individuos que cambiaron de lugar de residencia en los últimos cinco años tomó alguna decisión 
inconsistente, mientras que solo el $12 \%$ de las personas no desplazadas lo hicieron $(\mathrm{p}(\mathrm{Z}>\mathrm{z})=0,029)$.

Cuadro 3

Reversión de preferencias temporales

\begin{tabular}{|c|c|c|c|}
\hline & $\begin{array}{l}\text { Proporción de } \\
\text { participantes que } \\
\text { tomó decisiones } \\
\text { inconsistentes }\end{array}$ & Error estándar & $\mathrm{N}$ \\
\hline \multicolumn{4}{|l|}{ Nivel educativo } \\
\hline Secundaria incompleta o menos & $24,4 \%$ & 0,064 & 45 \\
\hline Secundaria completa o más & $4,8 \%$ & 0,046 & 21 \\
\hline Diferencia & $\begin{array}{r}19,7 \% \\
\mathrm{p}(Z>z)=0,027\end{array}$ & 0,079 & \\
\hline \multicolumn{4}{|c|}{ Cambió de residencia en los últimos 5 años } \\
\hline Sí & $31,6 \%$ & 0,049 & 19 \\
\hline No & $11,6 \%$ & 0,107 & 43 \\
\hline Diferencia & $\begin{array}{r}20 \% \\
\mathrm{p}(Z>z)=0,029\end{array}$ & 0,117 & \\
\hline
\end{tabular}

Nota: la prueba estadística es la de igualdad de medias (t estadístico). El tamaño de la muestra puede variar en caso de no tener toda la información del encuestado.

Fuente: elaboración propia.

Cuadro 4

Elecciones temporalmente inconsistentes

Estimaciones logística y logística exacta

\begin{tabular}{|c|c|c|c|c|}
\hline \multirow[b]{2}{*}{ Variable independiente } & \multicolumn{2}{|c|}{ Estimación logística } & \multicolumn{2}{|c|}{ Estimación logística exacta } \\
\hline & $\begin{array}{l}\text { Oportunidad } \\
\text { relativa }\end{array}$ & $\begin{array}{l}\text { Efecto } \\
\text { marginal }\end{array}$ & $\begin{array}{l}\text { Oportunidad } \\
\text { relativa }\end{array}$ & $\begin{array}{l}\text { Efecto } \\
\text { promedio }\end{array}$ \\
\hline Terminó bachillerato & $\begin{array}{c}-0,14^{*} \\
(0,145)\end{array}$ & $\begin{array}{l}-0,23^{* * *} \\
(0,109)\end{array}$ & $\begin{array}{r}0,15^{*} \\
(0,161)\end{array}$ & $-0,082$ \\
\hline $\begin{array}{l}\text { Cambió de residencia en } \\
\text { los últimos } 5 \text { años }\end{array}$ & $\begin{array}{r}4,30^{* * *} \\
(3,084) \\
\end{array}$ & $\begin{array}{r}0,17^{*} \\
(0,089) \\
\end{array}$ & $\begin{array}{r}4,11^{*} \\
(2,931) \\
\end{array}$ & 0,042 \\
\hline $\begin{array}{l}\mathrm{N}=62 \\
\text { Wald } \chi^{2}=8,22 ; \mathrm{p}=0,0164 \\
\text { Pseudo } \mathrm{R}^{2}=0,1387 \\
\text { (1) }\end{array}$ & & & \multicolumn{2}{|c|}{$\begin{array}{l}\mathrm{N}=62 \\
\text { Puntaje } \chi^{2}=7,239 ; \mathrm{p}=0,0182 \\
(2)\end{array}$} \\
\hline
\end{tabular}

Nota: variable dependiente: probabilidad de que el individuo haya tomado una decisión temporalmente inconsistente. Los errores estándar robustos van entre paréntesis. *: significativo al $10 \%$; **: significativo al $5 \%$; ${ }^{* * *}$ : significativo al $1 \%$.

(1) El efecto marginal se estimó asumiendo que las variables independientes toman su valor promedio.

(2) Efecto promedio $=\left[\left(\mathrm{p} / \mathrm{x}_{\mathrm{i}}=1 ; \mathrm{x}_{-\mathrm{i}}=0\right)+\left(\mathrm{p} / \mathrm{x}_{\mathrm{i}}=1 ; \mathrm{x}_{-\mathrm{i}}=1\right)\right] / 2-\left[\left(\mathrm{p} / \mathrm{x}_{\mathrm{i}}=0 ; \mathrm{x}_{-\mathrm{i}}=0\right)+\left(\mathrm{p} / \mathrm{x}_{\mathrm{i}}=0 ; \mathrm{x}_{-\mathrm{i}}=1\right)\right] / 2$.

Fuente: elaboración propia.

El cuadro 4 muestra los resultados de una estimación logit en la que la variable independiente indica si el individuo tomó alguna decisión temporalmente inconsistente. Estos resultados sugieren que, en promedio, una persona que no terminó bachillerato tiene una probabilidad 23 puntos porcentuales más alta de tomar una decisión temporalmente inconsistente, mientras que una persona recién desplazada tiene una probabilidad 17 puntos porcentuales más alta. Es 
posible que estos participantes dieran respuestas inconsistentes porque no entendieron las instrucciones, en parte porque tienen mayores dificultades para pensar problemas de naturaleza abstracta. Sin embargo, no podemos descartar que algunos participantes respondieran el cuestionario guiándose por heurísticas de decisión que no podemos racionalizar mediante los instrumentos propuestos.

\section{TASA DE DESCUENTO TEMPORAL REVELADA}

E1 cuadro 5 resume los resultados del ejercicio de preferencia temporal, para lo cual eliminé respuestas inconsistentes. Esto significó una reducción importante de la muestra, pero hace el análisis de los resultados menos ruidoso. $\mathrm{E} 1$ indicador de preferencia temporal es de carácter ordinal, en una escala de 0 a 10 según la línea en la que el participante haya preferido el pago futuro. Por lo tanto, la persona revela una tasa de descuento más alta cuanto más alto sea su puntaje.

a. Edad. La literatura es ambigua en cuanto a la relación que existe entre la edad y la tasa de descuento temporal. Harrison et al. (2002) encontraron evidencia a favor de la hipótesis del ingreso permanente en la población danesa: aunque los jóvenes tienden a tener una tasa de descuento alta, esta empieza a bajar con el paso del tiempo y luego nuevamente a aumentar una vez las personas llegan a edades cercanas o posteriores al retiro. Bauer et al. (2010) llegan a la misma conclusión para el caso de Uganda. Por otra parte, Kirby et al. (2002) hallaron que la tasa de descuento de los indígenas Tsimani en Bolivia aumentaba con la edad.

Para analizar los resultados de nuestro estudio dividí la población en tres grupos etarios: menores de 20 años (3 personas), entre 21 y 40 años ( 22 personas) y mayores de 40 años ( 29 personas). Si la hipótesis del ingreso permanente se cumple, las personas del segundo grupo deberían exhibir un indicador de preferencia temporal menor que el de los otros grupos. Aunque la mediana del indicador de preferencia temporal (1) para este grupo es menor que para el resto de la población (3), esta diferencia no es estadísticamente significativa (Wilcoxon-Mann-Whitney z=1,025; $\mathrm{p}=0,301$ ).

b. Nivel educativo. Los estudios de Kirby et al. (2002) y Harrison et al. (2002) encontraron que el nivel educativo y el alfabetismo están inversamente correlacionados con la tasa de descuento temporal. Esto es coherente con la teoría de Becker y Mulligan (1997) según la cual las inversiones en educación son al mismo tiempo inversiones en reducir la preferencia temporal, pues la educación facilita imaginar escenarios futuros. Sin embargo, la causalidad inversa también es 
razonable: los individuos más pacientes tienen un mejor desempeño escolar y alcanzan un mayor nivel educativo. Castillo et al. (2011), por ejemplo, encontraron que los niños más impacientes tienen una mayor probabilidad de tener problemas disciplinarios en el futuro. Con el fin de aislar el efecto causal de la educación en la tasa de descuento temporal, Bauer et al. (2010) explotaron las variaciones regionales a lo largo del tiempo en el acceso a la educación en Uganda, lo que les permitió concluir que los individuos que han tenido mejor acceso a la educación son más pacientes.

En nuestro estudio, los individuos que terminaron el bachillerato son en realidad más impacientes que aquellos que no lo hicieron: La mediana del indicador de preferencia temporal para el primer grupo es de 3.5 mientras que la mediana para el segundo grupo es de 1 . Sin embargo, esta diferencia no es estadísticamente significativa (WilcoxonMann-Whitney $\mathrm{z}=-1,043, \mathrm{p}=0,297)$.

Cuadro 5

Factores asociados al indicador de preferencia temporal

\begin{tabular}{|c|c|c|c|}
\hline & $\begin{array}{c}\text { Mediana del } \\
\text { indicador de } \\
\text { preferencia } \\
\text { temporal }\end{array}$ & $\begin{array}{l}\text { Test no paramétrico de rango } \\
\text { (Wilcoxon-Mann-Whitney) }\end{array}$ & $\mathrm{N}$ \\
\hline \multicolumn{4}{|l|}{ Edad } \\
\hline De 20 a 40 años & 1 & $\mathrm{z}=1,025$ & 22 \\
\hline Otro grupo etario & 3 & $\mathrm{p}=0,306$ & 32 \\
\hline \multicolumn{4}{|l|}{ Nivel educativo } \\
\hline Secundaria incompleta o menos & 1 & $z=-1,043$ & 34 \\
\hline Secundaria completa o más & 3,5 & $\mathrm{p}=0,297$ & 20 \\
\hline \multicolumn{4}{|l|}{$\begin{array}{l}\text { Cambió de residencia en los últimos } \\
5 \text { años }\end{array}$} \\
\hline Sí & 4 & $z=-1,749$ & 13 \\
\hline No & 1 & $p=0,08$ & 38 \\
\hline
\end{tabular}

Nota: el tamaño de la muestra puede variar en caso de no tener toda la información del encuestado.

Fuente: elaboración propia.

c. Ingresos y desplazamiento. Voors et al. (2012) hallaron que las personas expuestas al conflicto armado en Burundi muestran tasas de descuento temporal mayores que las del resto de la población. La estrategia empírica que usaron estos autores les permitió además concluir que el efecto es causal: la violencia, las guerras y las catástrofes naturales pueden alterar las preferencias individuales.

Aunque no tenemos información detallada de su nivel de exposición al conflicto armado, ni de las razones del desplazamiento, una proporción importante de la población de Barranca de Upía llegó 
al pueblo recientemente. La mediana del indicador de preferencia temporal para aquellas personas pertenecientes a hogares que llegaron al pueblo en los últimos cinco años es 4 , mientras que para el resto de la muestra es 1 (Wilcoxon-Mann-Whitney $z=-1,749, p=0,08$ ). Si bien estos resultados son compatibles con los hallazgos de Voors et al., también pueden ser explicados por la relación inversa, ampliamente documentada, entre nivel de ingresos y/o activos y tasa de descuento temporal (Harrison et al., 2002). En efecto, las personas desplazadas recientemente se encuentran en una situación más precaria que el resto de la población.

Para entender mejor cómo se relacionan estas variables con la probabilidad de revelar cierta preferencia temporal estimé un modelo logístico ordenado. Los resultados se resumen en el cuadro 6. La edad (pertenecer al grupo de 20 a 40 años) y el cambio de lugar de residencia son estadísticamente significativos. Las personas que hacen parte de este grupo etario tienen una probabilidad de tomar la elección más paciente 10 puntos porcentuales mayor. Por otro lado, los individuos que llegaron recientemente al municipio tienen una probabilidad de tomar la decisión más paciente 13 puntos porcentuales menor. Cabe aclarar que en este estudio no es posible identificar las razones por las cuales las personas desplazadas tienen indicadores de preferencia temporal más altos. Si bien puede ser por la influencia de la exposición al conflicto armado sobre las preferencias temporales, también es razonable pensar que el desplazamiento indica unas condiciones económicas más difíciles.

Cuadro 6

Indicador de preferencia temporal

Estimación logística ordenada

\begin{tabular}{lcc}
\hline Variable independiente & \multicolumn{2}{c}{ Estimación logística } \\
\cline { 2 - 3 } & Oportunidad relativa & $\begin{array}{c}\text { Efecto marginal sobre la } \\
\text { probabilidad de tomar la elección } \\
\text { más paciente }\end{array}$ \\
\hline De 20 a 40 años & $0,386^{*}$ & $0,096^{*}$ \\
Terminó bachillerato & $(0,205)$ & $(0,057)$ \\
Cambió de residencia en los últimos 5 años & 1,388 & $-0,033$ \\
& $(0,716)$ & $(0,053)$ \\
& $3,748^{* *}$ & $-0,134^{*}$ \\
& $(2,308)$ & $(0,069)$
\end{tabular}

Nota: el efecto marginal se estimó asumiendo que las variables independientes toman su valor promedio. Los errores estándar robustos van entre paréntesis. *: significativo al 10\%; **: significativo al 5\%; ***: significativo al $1 \%$.

Fuente: elaboración propia. 


\section{AVERSIÓN AL RIESGO}

Para analizar la aversión al riesgo uso una escala ordinal que va de 1 a 6 , donde 1 es la lotería segura y 6 la lotería más riesgosa. Cerca del 40\% de los 68 participantes prefirieron el pago seguro de $\$ 7.000$, mientras que los restantes se inclinaron por una de las otras loterías. El cuadro 7 resume los factores asociados a la aversión al riesgo.

a. Edad. En la línea de otros estudios previos, Cárdenas et al. (2013) y Rieger et al. (2013) encontraron que la edad está asociada a la aversión al riesgo, especialmente al riesgo por ganancias. En nuestro estudio no encontramos una asociación monotónica entre el indicador de aversión al riesgo y la edad (Spearman $\mathrm{Rho}=0,0626 ; \mathrm{p}=0,6261$ ).

b. Sexo. Al igual que en otros estudios anteriores encontramos que en promedio las mujeres son significativamente más aversas al riesgo: La mediana del indicador de aversión al riesgo es de 1 en el caso de las mujeres y 3 en el caso de los hombres (Wilcoxon-Mann-Whitney $\mathrm{z}=$ $-2,864 ; \mathrm{p}=0,0042)$.

c. Violencia y desplazamiento. En su estudio sobre aversión al riesgo en Afganistán, Callen et al. (2014) implementaron una serie de preguntas hipotéticas a una muestra de alrededor de 800 individuos, distribuidos en diferentes distritos electorales. E1 cuestionario incluía una pregunta de priming, en la que algunos individuos tenían que recordar un hecho que les hubiera producido miedo. También había ejercicios en los que los encuestados debían comparar diferentes loterías con el fin de que los investigadores pudieran establecer las primas de certeza individuales. Los autores encontraron que, aunque ni la memoria de un hecho productor de miedo ni la exposición a la violencia determinan independientemente los niveles de las primas de certeza, los dos factores combinados están asociados a la toma de decisiones más conservadoras.

Para el caso de Colombia, Moya (2016) encontró que niveles de exposición más severa a la violencia generan mayores niveles de aversión al riesgo por las ganancias. El canal que propone el autor, basándose en hallazgos de la psicología experimental, es que tal exposición a la violencia eleva los niveles de ansiedad.

Estos resultados son opuestos a los hallazgos de Voors et al. (2012), quienes estudiaron la relación entre exposición al conflicto armado y aversión al riesgo en Burundí, haciendo uso de experimentos con pagos reales. Los investigadores encontraron que la exposición al conflicto armado reduce la aversión al riesgo por las ganancias, aunque no tiene ningún efecto sobre la aversión al riesgo en las pérdidas. 
En nuestro estudio la mediana del indicador de aversión al riesgo para las personas recientemente desplazadas es igual que para el resto de la muestra (Wilcoxon-Mann-Whitney $z=0,688 ; p=0,4914$ ).

Cuadro 7

Factores asociados al indicador de aversión al riesgo

\begin{tabular}{lccc}
\hline Edad & Spearman Rho=0,0626; $\mathrm{p}=0,6261 ; \mathrm{N}=63$ & \\
\hline & $\begin{array}{c}\text { Mediana del } \\
\text { indicador de } \\
\text { aversión al riesgo }\end{array}$ & $\begin{array}{c}\text { Test no paramétrico } \\
\text { de rango } \\
\text { (Wilcoxon- } \\
\text { Mann-Whitney) }\end{array}$ & $\mathrm{N}$ \\
\hline $\begin{array}{l}\text { Sexo } \\
\text { Femenino }\end{array}$ & 1 & $\mathrm{z}=-2,864$ & 47 \\
Masculino & 3 & $\mathrm{p}=0,0042$ & 20 \\
Cambió de residencia en los últimos 5 5 años & 2 & $\mathrm{z}=-0,688$ & 19 \\
Sí & 2 & $\mathrm{p}=0,4914$ & 44 \\
No & & &
\end{tabular}

Fuente: elaboración propia.

El cuadro 8 resume los resultados de la estimación de un modelo logístico ordenado en donde la variable dependiente es el indicador de aversión al riesgo. Controlando por la edad y el sexo, las personas recientemente desplazadas tienen una probabilidad 28 puntos porcentuales más baja de tomar la decisión más conservadora. Este resultado, significativo a un nivel de confianza del 10\%, implica que las personas expuestas al conflicto armado o con peores condiciones económicas están más dispuestas a escoger las loterías riesgosas. Por otro lado, controlando por los otros factores, los hombres tienen una probabilidad 47 puntos porcentuales más baja de tomar la decisión más segura.

Cuadro 8

Indicador de aversión al riesgo: estimación logística ordenada

\begin{tabular}{lcc}
\hline Variable independiente & $\begin{array}{c}\text { Epstimación logística } \\
\text { relativa }\end{array}$ & $\begin{array}{c}\text { Efecto marginal sobre la } \\
\text { probabilidad } \\
\text { de tomar la elección más } \\
\text { conservadora }\end{array}$ \\
\hline Edad & 0,987 & 0,0031 \\
Sexo masculino & $(0,017)$ & $(0,004)$ \\
Cambió de residencia en los últimos 5 años & $7,025^{* * *}$ & $-0,468^{* * *}$ \\
& $(3,934)$ & $(0,137)$ \\
$\mathrm{N}=63$; Wald $\chi^{2}=13,5 ; \mathrm{p}=0,0037 ;$ Pseudo $\mathrm{R}^{2}=0,0639$ & $(1,928)$ & $(0,146)$ \\
\hline
\end{tabular}

Nota: el efecto marginal fue estimado asumiendo que las variables independientes toman su valor promedio. Los errores estándar robustos van entre paréntesis. *: significativo al 10\%; **: significativo al $5 \%$; ${ }^{* * *}$ : significativo al $1 \%$.

Fuente: elaboración propia. 


\section{CONCLUSIONES}

Este artículo presenta evidencia exploratoria de algunos factores correlacionados con las preferencias económicas en Colombia. Estos resultados se obtuvieron mediante una encuesta de hogares y un experimento de campo en Barranca de Upía.

En concordancia con algunos hechos estilizados de la literatura sobre el tema se encuentra que 1) la edad está correlacionada con las preferencias temporales, quizá conforme a la hipótesis del ingreso permanente, y 2) la aversión al riesgo está correlacionada con el sexo; las mujeres son más aversas al riesgo, incluso controlando factores como la edad y el desplazamiento.

El resultado más relevante es que las personas que llegaron al pueblo en los últimos cinco años, bien sea por razones económicas o por el conflicto armado, presentan patrones de preferencias económicas distintas al del resto de la población. Controlando la edad y el nivel educativo, estas personas exhiben niveles más altos de preferencia temporal. Y controlando la edad y el sexo, tienen niveles de aversión al riesgo más bajos que los del resto de la población.

Este estudio tiene tres grandes limitaciones: 1) el tamaño de la muestra solo permite ver las correlaciones y los efectos de gran magnitud, 2) no permite hacer inferencias causales ni establecer los mecanismos por los cuales ciertos grupos tienen preferencias diferentes, y 3) aunque en la encuesta de hogares se siguió un procedimiento aleatorio estándar y es representativa de la zona urbana del pueblo, la sub muestra utilizada para el experimento tuvo sesgos de selección; el más importante es que solo invitamos personas que supieran leer y escribir. Esto limita la validez externa de los hallazgos. Sin embargo, la sub muestra es variable en factores clave como la edad, el sexo, el nivel de ingresos y el desplazamiento.

El estudio reitera que las personas recién desplazadas son un grupo especial, no solo porque tienen niveles de pobreza más altos o sufrieron despojo de tierras, sino porque sus preferencias económicas son diferentes de las del resto de la población. Por tanto, el impacto del conflicto, la violencia y las catástrofes naturales sobre las preferencias individuales se debe investigar con mayor profundidad para contribuir al diseño de políticas públicas en el post conflicto. 


\section{ANEXO 1}

Invitación n. :

Supongamos ahora que Ud. gana un premio de $\$ 10.000$ para ser entregado mañana viernes 20 de febrero. Si Ud. acepta recibir este dinero de mañana en una semana, o sea el viernes 27 de febrero, recibirá un valor igual o superior. ¿¿Ud. qué prefiere? Marque una $x$ en la casilla que está al lado de su opción preferida.

Decisión 1

$\$ 10.000$ mañana

Decisión 2

$\$ 10.000$ mañana $\square$

Decisión 3

$\$ 10.000$ mañana $\square$

Decisión 4

$\$ 10.000$ mañana

Decisión 5

$\$ 10.000$ mañana $\square$

Decisión 6

$\$ 10.000$ mañana

Decisión 7

$\$ 10.000$ mañana

Decisión 8

$\$ 10.000$ mañana $\square$

Decisión 9

\$10.000 mañana

Decisión 10

$\$ 10.000$ mañana $\square$

\$10.000 de mañana en ocho días

$\$ 11.000$ de mañana en ocho días $\square$

\$12.000 de mañana en ocho días

\$13.000 de mañana en ocho días

$\$ 14.000$ de mañana en ocho días $\square$

$\$ 15.000$ de mañana en ocho días $\square$

\$16.000 de mañana en ocho días

\$17.000 de mañana en ocho días

$\$ 18.000$ de mañana en ocho días $\square$

\$19.000 de mañana en ocho días

\section{ANEXO 2}

Invitación n. :

En la bolsa tenemos una bola blanca y una bola negra. Vamos a sacar una de las dos bolas al azar. Abajo tenemos 6 juegos donde Ud. puede ganar una cantidad diferente según la bola que salga. ¿Qué juego le gustaría jugar? Márquelo con una X en la casilla al lado del título

\section{Juego 1}

O Pierdo $\$ 3.000 \bigcirc$ Gano $\$ 7.000$

\section{Juego 2}

○ Pierdo $\$ 4.500 \bigcirc$ Gano $\$ 10.000$ 
Juego 3

○ Pierdo $\$ 6.000$ ○ Gano $\$ 13.000$

Juego 4

O Pierdo $\$ 7.500$ ○ Gano $\$ 16.500$

Juego 5

○ Pierdo $\$ 9.000 \bigcirc$ Gano $\$ 19.000$

Juego 6

○ Pierdo $\$ 10.000 \bigcirc$ Gano $\$ 20.000$

\section{REFERENCIAS BIBLIOGRÁFICAS}

1. Andersen, S. et al. (2008). Eliciting risk and time preferences. Econométrica, 76(3), 583-618.

2. Anderson, C. L. et al. (2004). Discount rates in Vietnam. Economic Development and Cultural Change, 52(4), 873-887.

3. Andreoni, J. y Sprenger, C. (2012). Estimating time preferences from convex budgets. American Economic Review, 102(7), 3333-3356.

4. Arnaud, R. y Couture, S. (2012). Stability of risk preference measures: results from a field experiment on French farmers. Theory and Decision, 73(2), 203-221.

5. Bauer, M.; Chytilov, J. et al. (2012). Behavioral foundations of microcredit: experimental and survey evidence from rural India. American Economic Review, 102(2), 1118-1139.

6. Bauer, M. y Chytilov, J. (2010). The impact of education on subjective discount rate in Ugandan villages. Economic Development and Cultural Change, 58(4), 643-669.

7. Becker, G. S. y Mulligan, C. B. (1997). The endogenous determination of time preference. The Quarterly Journal of Economics, 112(3), 729-758.

8. Berg, J.; Dickhaut, J. et al. (1995). Trust, reciprocity, and social history. Games and economic behavior, 10(1), 122-142.

9. Brick, K.; Visser, M. et al. (2012). Risk aversion: Experimental evidence from South African fishing communities. American Journal of Agricultural Economics, 94(1), 133-152.

10. Callen, M. et al. (2014). Violence and risk preference: Experimental evidence from Afghanistan. American Economic Review, 104(1), 123-148.

11. Cárdenas, J. C. y Carpenter, J. (2008). Behavioural development economics: Lessons from field labs in the Developing World. Journal of Development Studies, 44(3), 311-338.

12. Cárdenas, J. C. y Carpenter, J. (2013). Risk attitudes and economic well-being in Latin America. Journal of Development Economics, 103, 52-61. 
13. Carlsson, F. et al. (2012). Household decision making in rural China: Using experiments to estimate the influences of spouses. Journal of Economic Behavior E Organization, 84(2), 525-536.

14. Charness, G.; Gneezy, U. et al. (2013). Experimental methods: Eliciting risk preferences. Journal of Economic Behavior E Organization, 87, 43-51.

15. Cook, J. et al. (2013). Measuring risk aversion among the urban poor in Kolkata, India. Applied Economics Letters, 20(1), 1-9.

16. Dave, C. et al. (2010). Eliciting risk preferences: When is simple better? Journal of Risk and Uncertainty, 41(3), 219-243.

17. Dufwenberg, M.; Gächter, S. et al. (2011). The framing of games and the psychology of play. Games and Economic Behavior, 73(2), 459-478.

18. Eckel, C. C. y Grossman, P. J. (2008). Differences in the economic decisions of men and women: Experimental evidence. Handbook of Experimental Economics Results 1, chap. 57, 509-519.

19. Fehr, E. y Gächter, S. (2000). Fairness and retaliation: The economics of reciprocity. The Journal of Economic Perspectives, 14(3), 159-181.

20. Fischbacher, U. y Föllmi-H. F. (2013). Lies in disguise - An experimental study on cheating. Journal of the European Economic Association, 11(3), 525-547.

21. Gaudecker, H.-M.; Van, S. A. et al. (2012). Experts in experiments: How selection matters for estimated distributions of risk preferences. Journal of Risk and Uncertainty, 45(2), 159-190.

22. Hans, P. B. (1980). Attitudes toward risk: Experimental measurement in rural India. American Journal of Agricultural Economics, 62(3), 395-407.

23. Harrison, G. W.; Lau, M. I. et al. (2002). Estimating individual discount rates in Denmark: A field experiment. American Economic Review, 92(5), 1606-1617.

24. Johnson, M. W. y Bickel, W. K. (2002). Within-subject comparison of real and hypothetical money rewards in delay discounting. Journal of the Experimental Analysis of Behavior, 77(2), 129-146.

25. Kirby, K. N. et al. (2002). Correlates of delay-discount rates: Evidence from Tsimane' Amerindians of the Bolivian rain forest. Journal of Economic Psychology, 23(3), 291-316.

26. Kirby, K. N. y Herrnstein, R. J. (1995). Preference reversals due to myopic discounting of delayed reward. Psychological Science, 6(2), 83-89.

27. Kirby, K. N.; Winston, G. C. et al. (2005). Impatience and grades: Delay-discount rates correlate negatively with college GPA. Learning and Individual Differences, 15(3), 213-222.

28. Liu, E. M. y Huang, J. (2013). Risk preferences and pesticide use by cotton farmers in China. Journal of Development Economics, 103, 202-215.

29. Meier, S. y Sprenger, C. (2010). Present-biased preferences and credit card borrowing. American Economic Journal: Applied Economics, 2(1), 193-210.

30. Meier, S. y Sprenger, C. D. (2013). Discounting financial literacy: Time preferences and participation in financial education programs. Journal of Economic Behavior E Organization, 95, 159-174. 
31. Mosley, P. y Verschoor, A. (2005). Risk attitudes and the vicious circle of poverty. European Journal of Development Research, 17(1), 59-88.

32. Moya, A. (2016). Violence, psychological trauma, and induced changes in risk attitudes: Evidence from victims of violence in Colombia, working paper, 2016.

33. Rieger, M. O.; Wang, M. et al. (2015). Risk preferences around the world. Management Science, 61(3), 637-648.

34. Shane, F.; Loewenstein, G. et al. (2002). Time discounting and time preference: A critical review. Journal of Economic Literature, 40(2), 351-401.

35. Sutter, M. et al. (2013). Impatience and uncertainty: Experimental decisions predict adolescents' field behavior. American Economic Review, 103(1), 510-531.

36. Tanaka, T.; Camerer, C. F. et al. Risk and time preferences: Linking experimental and household survey data from Vietnam. American Economic Review, 100(1), 557-571.

37. Voors, M. J. et al. (2012). Violent conflict and behavior: A field experiment in Burundi. American Economic Review, 102(2), 941-964. 